ORIGINAL ARTICLE

\title{
Vegetarian Diets and Cardiovascular Risk in Women
}

\author{
Bianca Oliveira, ${ }^{(1)}$ Luciana Nicolau Aranha, ${ }^{(0)}$ Priscila dos Santos Gomes Olivares, ${ }^{(0)}$ Tamira Guilherme Rocha \\ Negrão, ${ }^{(1)}$ Glorimar Rosa, ${ }^{(1)}$ Gláucia Maria Moraes de Oliveira \\ Universidade Federal do Rio de Janeiro, Rio de Janeiro - RJ - Brazil
}

\section{Abstract}

Background: Vegetarian diets have favorable effects on cardiovascular risk, provided that they do not contain ultra-processed foods (UPF).

Objective: To compare the metabolic profile, cardiovascular risk, body composition, and food consumption in vegan (VEG), lacto-ovo vegetarian (LOV), and omnivorous (OMNI) women. To verify the association between UPF consumption and cardiovascular risk.

Methods: Cross-sectional study with 119 VEG $(\mathrm{n}=43)$, LOV $(\mathrm{n}=38)$, and OMNI $(\mathrm{n}=38)$ women. Anthropometric and biochemical parameters and the Framingham risk score were assessed. Food consumption was assessed by means of a 3-day food register, and intake of macronutrients, micronutrients, and UPF was estimated. The correlation between UPF consumption and cardiovascular risk was assessed using Spearman's coefficient, with a significance level of 5\%.

Results: The groups showed low cardiovascular risk, without significant difference between them. The VEG and LOV groups had lower body mass index, neck circumference, body shape index, and systolic blood pressure ( $\mathrm{p}$ $<0.05$ ) than the OMNI group; greater consumption of carbohydrates, sugars, dietary fibers, micronutrients, betacarotene, and carotenoids; and lower consumption of total fat, saturated fatty acids, and cholesterol ( $\mathrm{p}<0.05)$. Consumption of UPF was lower in the LOV group (5.7 [0.0- 19.8]) than in the OMNI group (14.9 [5.1 - 22.3 ]; $\mathrm{p}<$ 0.05). UPF consumption was associated with SBP $(\varrho=0.439 ; \mathrm{p}=0.007)$ and blood sugar $(\varrho=0.422 ; \mathrm{p}=0.010)$ in the VEG group, and in the LOV group it was inversely associated with LDL-c ( $\varrho=-0.456 ; \mathrm{p}=0.010)$.

Conclusion: Vegetarian women showed better body composition and dietary quality than OMNI women. It is important to take consumption of UPF in vegetarians into consideration, in order to improve cardiovascular risk in women.

Keywords: Vegetarian Diet; Vegan; Women; Risk Factors; Industrialized Foods.

\section{Introduction}

Worldwide prevalence of cardiovascular disease (CVD) practically doubled from 271 million (95\% uncertainty interval [UI]: 257 to 285 million) in 1990 to 523 million (95\% UI: 497 to 550 million) in 2019, and the number of deaths due to CVD increased constantly from 12.1 million (95\% UI: 11.4 to 12.6 million) in 1990 to 18.6 million (95\% UI: 17.1 to 19.7 million) in $2019 .{ }^{1}$ In young women, an increase has been observed in hospitalizations due to CVD and acute myocardial infarction, which has occurred mainly due to an increase in the prevalence of obesity and cardiometabolic risk factors. ${ }^{2}$ In Brazil, according to

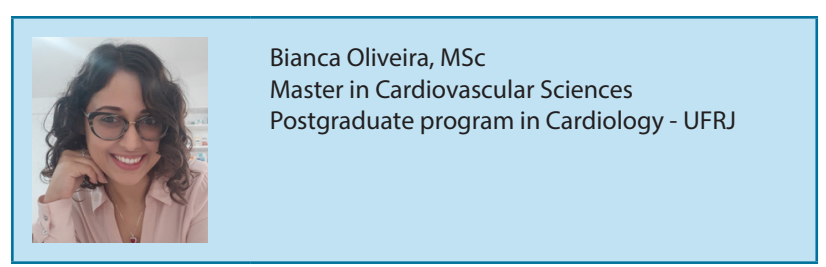


data from the Informatics Department of the Unified Health System (DATASUS, acronym in Portuguese), in $2019,17.2 \%$ of deaths that occurred in women of childbearing age were due to circulatory system diseases, thus representing the second leading cause of mortality in this group. ${ }^{3}$

Considering that the majority of cardiometabolic risk factors do not occur with clinical manifestations, early identification can be important in order to modify prognosis of CVD. Accordingly, some tools have been suggested to predict cardiovascular risk, such as the Framingham Risk Score (FRS), which assesses short-term risk of coronary artery disease (CAD), and traditional anthropometric indices, such as body mass index (BMI), waist circumference (WC), and waist-to-height ratio (WHR), as well as new indices, such as lipid accumulation product (LAP), body roundness index (BRI), and body shape index (ABSI). ${ }^{4,5}$

In relation to strategies for modifying the course of CVD, diet plays a crucial role. Different dietary patterns (DP) such as the Dietary Approaches to Stop Hypertension (DASH) eating plan, the Mediterranean $\mathrm{DP}$, and the vegetarian DP have been proposed by the American Heart Association/American College of Cardiology (AHA/ACC) both for prevention and treatment of CVD and in general, emphasizing the increased consumption of vegetables, fruits, whole grains, and legumes and limited intake of red meat, sweets, sugar-sweetened beverages, and salty or highly processed foods. ${ }^{6}$

In recent years, the number of vegetarians has increased, and this DP has been associated with health benefits, given that it involves reduced cardiometabolic risk factors, and it may contribute to a lower prevalence of CVD. ${ }^{7-9}$ The benefits of the vegetarian DP result from increased consumption of vegetables, sources of fiber, and phytonutrients, which reduce inflammation and oxidative stress, providing cardiovascular protection. ${ }^{10}$

Although plant-based diets have been associated with lower risk of CVD, they may not always have beneficial health effects, in the event that they are rich in unhealthy plant foods (sugar-sweetened juices/beverages, refined grains, fried potatoes, sweets) $\cdot{ }^{11}$ It is known that consumption of ultra-processed foods (UPF) is associated with the main cardiovascular risk factors, such as obesity, hypertension, dyslipidemia, hyperglycemia, and hyperinsulinemia; reduced consumption of these foods is, therefore, recommended. ${ }^{12}$ Few studies in the literature have investigated the consumption of UPF in vegetarians, especially in women. ${ }^{13,14}$

It is important to comprehend the nutritional profile of women and to compare different DPs in order to verify their associations with diseases, such as CVD, thus establishing nutritional behavior protocols for prevention. In this manner, the objective of this study was to compare the metabolic profile, cardiovascular risk, body composition, and food consumption in women who adhered to vegan (VEG), lacto-ovo-vegetarian (LOV), and omnivorous (OMNI) diets, as well as to verify consumption of UPF.

\section{Individuals And Methods}

\section{Study Groups}

This cross-sectional study was conducted between January and July 2019, with a convenience sample of 119 women selected at a clinical nutrition outpatient clinic, in Rio de Janeiro. Women ages 20 to 59 years old were selected; they had adhered to the DP for at least 6 months, and they were divided into the following groups: VEG (no consumption of any products of animal origin), LOV (consumption of eggs, milk, and dairy products), and OMNI (consumption of red meat, fish, chicken, eggs, milk, and dairy products. Pregnant and breastfeeding women were excluded.

This study received approval from the Ethics Committee of the Clementino Fraga Filho University Hospital of the Federal University of Rio de Janeiro, and it was registered under number 89033118.1.0000.5257. All participants signed a free and informed consent form.

Anthropometric, body composition, blood pressure, and cardiovascular risk assessment

Anthropometric assessment was carried out, including measurements of body mass in $\mathrm{kg}$, height in $\mathrm{m}$, waist circumference (WC) in $\mathrm{cm}$, and neck circumference (NC) in cm. Body mass index (BMI) was calculated (weight/height ${ }^{2}$ ) and classified in accordance with the parameters established by the World Health Organization..$^{15}$ The waist-to-height ratio (WHR), ${ }^{16}$ visceral adiposity index (VAI) ${ }^{17} \mathrm{LAP},{ }^{18} \mathrm{BRI},{ }^{19}$ and ABSI were also calculated and classified. ${ }^{20}$

Body composition was assessed by tetrapolar bioimpedance (Biodynamics 450,biodynamics corporation, Washington), and body fat percentage 
was classified according to Lohman et al. ${ }^{21}$. Blood pressure was measured by the auscultatory method. ${ }^{22}$ Cardiovascular risk was calculated by means of the FRS, using the standardized calculator (https://qxmd.com/ calculate/calculator_253/framingham-risk-score-atp-iii), which takes the following variables into account: sex, age, total cholesterol, high-density lipoprotein (HDL), systolic blood pressure (SBP), use of medications for systemic arterial hypertension, and smoking. ${ }^{4}$

\section{Biochemical Assessment}

Blood samples were collected in the morning, after a 12hour fasting period, in tubes with gel to obtain serum; 30 minutes after collection, blood samples were centrifuged for 15 minutes at 4,000 rpm. Serum concentrations of glucose, triglycerides, HDL, and total cholesterol were determined by the enzymatic method, using an automated biochemical analyzer (Labtest Diagnostica SA, Vista Alegre, Lagoa Santa-Mg. Brazil). Low-density lipoprotein (LDL) concentrations were calculated using the Friedewald formula. ${ }^{23}$ Serum insulin concentration was obtained by chemiluminescence, and insulin resistance was estimated using the homeostasis model assessment of insulin resistance (HOMA-IR) index. ${ }^{24}$

Assessment of Food Consumption and Degree of Physical Activity

Consumption of energy, macronutrients, and micronutrients was assessed using a 3-day food register ( 2 typical and 1 atypical days), and data were analyzed using Food Processor software, version 7.2 (EshaResearch, Salem, MA, USA). Assessment of the adequacy of nutritional composition of micronutrients was based on Dietary Reference Intakes. ${ }^{25}$ Adequate consumption of fiber, saturated fat, and sodium was established based on the guidelines of the Brazilian Society of Cardiology. ${ }^{26}$ Consumption of processed foods and UPF was classified according to the NOVA classification, by Monteiro et al. ${ }^{27}$

Degree of physical activity was assessed by the short version of the International Physical Activity Questionnaire. ${ }^{28}$

\section{Statistical Analysis}

Statistical analyses were conducted using IBM ${ }^{\circledR}$ SPSS ${ }^{\circledR}$ Statistics software, version 25 (Armonk, NY, USA). Categorical variables were shown as percentages and analyzed using the chi-square $\left(\chi^{2}\right)$ test. Normality of variables was evaluated using the Kolmogorov-Smirnov method. Continuous variables were represented as median and interquartile range, because they did not show normal distribution. For comparison between groups, the Kruskal-Wallis test with post hoc Bonferroni was used. Spearman's correlation coefficients were used to evaluate correlations between percentage of total energy value from UPF and cardiovascular risk factors. Values were considered significant when $\mathrm{p}<0.05$.

\section{Results}

A total of 119 women were included in the study. Their characteristics are shown in Table 1 . Of these participants, 43 (36\%) were VEG, with median age of 29 (24 to 35) years; 38 (32\%) were LOV, with median age of 27.5 (22 to 36$)$ years; and 38 (32\%) were OMNI, with median age of 33.5 (27 to 40) years. The VEG and LOV group had higher level of schooling than the OMNI group.

The prevalence of excess body mass (overweight or obesity) in the study population was $25.6 \%$ in VEG, $26.3 \%$ in LOV, and $57.8 \%$ in OMNI. Even though the cutoff values of the anthropometric indices were within adequate values for all groups, VEG and LOV had lower BMI, NC, and ABSI compared to OMNI. Furthermore, SBP was significantly lower in the groups that adhered to vegetarian DPs (VEG and LOV). Moreover, the LOV group had significantly lower WHR, WC, BRI, and DBP values than the OMNI group, and LDL-c concentrations were lower in the VEG group than in the OMNI group (Table 2).

With the aim of assessing short-term risk of CAD, the FRS was calculated, and it showed that all study groups had low $(1 \%)$ risk of CAD in the short term ( $\leq 10$ years), without any statistically significant difference $(p=0.340)$.

When assessing food consumption, it was observed that VEG and LOV had higher consumption of carbohydrates; sugars; dietary fiber; vitamins A, C, and E; potassium; beta-carotene; and carotenoids, as well as lower consumption of total fat, saturated fatty acids, cholesterol, sodium, and $\mathrm{Na} / \mathrm{Kcal}$ ratio than the OMNI group. Moreover, UPF consumption was significantly lower in LOV (5.7 [0.0 - 19.8]) than in OMNI (14.9 [5.1 22.3]; $\mathrm{p}<0.05)$, as can be observed in Table 3 .

The correlation test between percentage of TEV from UPF and cardiovascular risk factors demonstrated that consumption of UPF was positively associated with SBP $(\varrho=0.439 ; \mathrm{p}=0.007)$ and blood sugar $(\varrho=0.422 ; \mathrm{p}=$ $0.010)$ in the VEG group and negatively associated with 
Table 1 - Sociodemographic characteristics according to type of diet adopted

\begin{tabular}{|c|c|c|c|c|}
\hline & $\begin{array}{l}\text { VEG } \\
(n+3)\end{array}$ & $\begin{array}{l}\text { LOV } \\
\text { (n 38) }\end{array}$ & $\begin{array}{l}\text { OMNI } \\
\text { (n 38) }\end{array}$ & $\mathrm{p}$ value \\
\hline Age (years) & $29(24-35)$ & $27.5(22-36)$ & $33.5(27-40)$ & 0.07 \\
\hline Per capita income (MW) & $0.03(0.001-1.2)$ & $0.7(0.001-2)$ & $1.5(0.4-2)$ & 0.20 \\
\hline \multicolumn{5}{|l|}{ Level of schooling (n, \%) } \\
\hline Primary & (0) - $0 \%$ & (1) $-2.6 \%$ & (6) $-15.8 \%$ & \multirow{3}{*}{0.001} \\
\hline Secondary & $(2)-4.7 \%$ & (3) $-7.9 \%$ & (11) $-28.9 \%$ & \\
\hline Tertiary & $(41)-95.3 \%$ & (34) $-89.5 \%$ & $(21)-55.3 \%$ & \\
\hline \multicolumn{5}{|l|}{ Skin color $(n-\%)$} \\
\hline Black & (4) $-9.3 \%$ & (2) $-5.3 \%$ & (7) $-18.4 \%$ & \multirow{3}{*}{0.15} \\
\hline Mixed & (13) $-30.2 \%$ & $(10)-26.3 \%$ & (15) $-39.5 \%$ & \\
\hline White & (26) $-60.5 \%$ & (26) $-68.4 \%$ & (16) $-42.1 \%$ & \\
\hline Duration of vegetarianism (years) & $3(1.2-5)$ & $2(1-4)$ & $0(0-0)$ & 0.10 \\
\hline Nutritional follow-up (n, \%) & (20) $-46.5 \%$ & (17) $-44.7 \%$ & (11) $-28.9 \%$ & 0.22 \\
\hline Alcohol use & $(22)-51.2 \%$ & (20) $-52.6 \%$ & $(10)-26.3$ & 0.06 \\
\hline Smoking & $(0)-0 \%$ & (2) $-5.3 \%$ & (2) $-5.3 \%$ & 0.08 \\
\hline $\mathrm{SAH}$ & $(0)-0 \%$ & (1) $-2.6 \%$ & $1-2.6 \%$ & 0.14 \\
\hline $\mathrm{DM}$ & $(0)-0 \%$ & $(0)-0 \%$ & (2) $-5.2 \%$ & 0.11 \\
\hline CVD & $(0)-0 \%$ & $(1)-2.6 \%$ & (0) - $0 \%$ & 0.17 \\
\hline \multicolumn{5}{|l|}{ Degree of physical activity (IPAQ) } \\
\hline Active (n, \%) & (33) $-76.6 \%$ & $(24)-63.2 \%$ & (23) $-62.5 \%$ & 0.58 \\
\hline Sedentary $(\mathrm{n}, \%)$ & (10) $-23.4 \%$ & (14) $-36.9 \%$ & (13) $-37.5 \%$ & \\
\hline \multicolumn{5}{|c|}{$\begin{array}{l}\text { Values shown as median and interquartile range and frequency (n, \%). Chi-square test for categorical variables. Kruskal-Wallis test with post hoc } \\
\text { Bonferroni for continuous variables. Values considered statistically significant: } p \text { < 0.05. CVD: cardiovascular disease; DM: diabetes mellitus; IPAQ: } \\
\text { International Physical Activity Questionnaire; LOV: lacto-ovo vegetarian; MW: minimum wage; OMNI: omnivorous; SAH: systemic arterial } \\
\text { hypertension; VEG: vegan. }\end{array}$} \\
\hline
\end{tabular}

LDL-c ( $\mathrm{Q}=-0.456 ; \mathrm{p}=0.010)$ in the LOV group, as can be observed in Table 4 . No associations were observed between UPF consumption and the FRS and 10-year risk of CVD in any of the groups.

The main results of this article can be seen in Figure 1.

\section{Discussion}

In this study, women who adhered to a vegetarian DP had better body composition and dietary quality, in comparison with the OMNI DP. In the VEG group, UPF consumption was associated with higher blood sugar and SBP.

Our sample was characterized by young women, who were apparently healthy, and the majority were active. Consequently, the FRS indicated low short-term risk of
CAD for all groups, and the biochemical parameters were within the limits of normality. Navarro et al. ${ }^{29}$ carried out a cross-sectional study, with 88 apparently healthy men (44 vegetarians and 44 omnivores), age $\geq 35$ years, and they observed FRS < 10; however, they found that risk of CAD, assessed by the FRS, was lower in vegetarians, as were some cardiovascular risk factors, suggesting that a plant-based diet could be considered protective for cardiovascular health.

The identification of individuals who are susceptible to developing CVD is extremely important and, at the same time, challenging, especially in those who are asymptomatic. The performance of scores for predicting cardiovascular risk varies considerably between populations, and evidence that supports the use of cardiovascular risk scores for primary 
Table 2 - Blood pressure, body composition, and biochemical data, according to type of diet adopted

\begin{tabular}{|c|c|c|c|}
\hline Variables & $\begin{array}{l}\text { VEG } \\
(n+3)\end{array}$ & $\begin{array}{l}\text { LOV } \\
\text { (n 38) }\end{array}$ & $\begin{array}{l}\text { OMNI } \\
\text { (n 38) }\end{array}$ \\
\hline BMI $\left(\mathrm{kg} / \mathrm{m}^{2}\right)$ & $22.3(20.2-25.2)$ & $22.2(20.9-25.2)$ & $25(22.4-28.9)^{a . b}$ \\
\hline Fat mass (\%) & $26.6(24-30.2)$ & $26.4(24.3-31.7)$ & $27(22.6-31.5)$ \\
\hline Lean mass (\%) & $73.2(69.4-75.8)$ & $73.6(67.9-75.7)$ & $71.2(66.0-76.5)$ \\
\hline WHR & $0.5(0.4-0.5)$ & $0.4(0.4-0.5)$ & $0.5(0.4-0.5)^{b}$ \\
\hline $\mathrm{WC}(\mathrm{cm})$ & $74(69-80.5)$ & $73.5(69-77.6)$ & $80.5(72.7-85.2)^{\mathrm{b}}$ \\
\hline $\mathrm{NC}(\mathrm{cm})$ & $31.5(30.4-33)$ & $32(30.9-33.1)$ & $33.6(31-36)^{a . b}$ \\
\hline VAI & $0.9(0.7-1.3)$ & $1.05(0.8-1.4)$ & $1.01(0.7-1.6)$ \\
\hline LAP & $12.8(7.04-19.2)$ & $13.9(8.2-22.2)$ & $17.2(10.9-29.8)$ \\
\hline $\mathrm{CI}$ & $1.1(1.08-1.2)$ & $1.1(1.07-1.2)$ & $1.1(1.07-1.2)$ \\
\hline ABSI & $0.6(0.5-0.6)$ & $0.5(0.5-0.6)$ & $0.5(0.4-0.5)^{a . b}$ \\
\hline BRI & $1.4(1.2-1.6)$ & $1.4(1.2-1.5)$ & $1.6(1.3-1.8)^{b}$ \\
\hline $\mathrm{SBP}(\mathrm{mmHg})$ & $110(100-110)$ & $105(100-110.5)$ & $115(110-120)^{\text {a.c }}$ \\
\hline $\mathrm{DBP}(\mathrm{mmHg})$ & $70(70-80)$ & $70(70-80)$ & $80(70-80)^{\mathrm{b}}$ \\
\hline Blood glucose (mg/dL) & $80(73-89)$ & $77(70.8-86)$ & $76(68-83)$ \\
\hline Insulin $(\mathrm{mIU} / \mathrm{mL})$ & $8(4.8-13)$ & $8(6.8-13.3)$ & $11(6.5-15)$ \\
\hline HOMA-IR & $1.7(0.75-2.7)$ & $1.6(1.1-2.4)$ & $2.1(1.3-3)$ \\
\hline Triglycerides (mg/dL) & $75(57-91)$ & $79.5(63-102.8)$ & $74.5(57.5-101)$ \\
\hline $\mathrm{TC}(\mathrm{mg} / \mathrm{dL})$ & $162(140-183)$ & $175.5(153.2-205.5)$ & $181(153-213.3)$ \\
\hline LDL-c (mg/dL) & $86(70-104)$ & $98(80.3-118.3)$ & $106(86.7-123.5)^{\mathrm{a}}$ \\
\hline HDL-c (mg/dL) & $59(49-68)$ & $62(47.8-70.3)$ & $59(47-67.3)$ \\
\hline \multicolumn{4}{|c|}{$\begin{array}{l}\text { Values shown as median and interquartile range. Kruskal-Wallis test with post hoc Bonferroni to analyze significance level. Values considered statistically } \\
\text { significant: } p<0.05 .{ }^{a} p<0.05 \text { between OMNI and VEG; }{ }^{b} p<0.05 \text { between OMNI and LOV; }{ }^{c} p \leq 0.001 \text { between OMNI and LOV. ABSI: body shape } \\
\text { index; BMI: body mass index; BRI: body roundness index; CI: conicity index; DBP: diastolic blood pressure; HDL: high-density lipoprotein; LAP: lipid } \\
\text { accumulation product; LDL: low-density lipoprotein; SBP: systolic blood pressure; TC: total cholesterol; TG: triglycerides; VAI: visceral adiposity index; } \\
\text { WC: waist circumference; WHR: waist-to-height ratio. }\end{array}$} \\
\hline
\end{tabular}

prevention is scarce. ${ }^{30}$ Furthermore, women have sexspecific factors (early menarche, hormonal factors, autoimmune conditions, pregnancy-associated factors, etc.), which have demonstrated association with increased cardiovascular risk. ${ }^{31}$ Accordingly, it is necessary to create new models in order to assess cardiovascular risk in this specific group and to determine the role of these scores in predicting cardiovascular risk in primary prevention.

When comparing DPs, studies have shown that vegetarians have lower BMI and WC than omnivores. ${ }^{9,32}$ In a cohort of 49,098 adults in Taiwan, a lower prevalence of overweight was observed in vegetarians than in nonvegetarians; the authors also found that, for each year on a vegan diet, the risk of obesity decreased by $7 \% .{ }^{33}$ We also found that omnivores had more overweight and greater WC, NC, WHR, ABSI, and BRI than vegetarians. New anthropometric indices have been used to assess the risk of CVD; the BRI, for example, is an index based on WC and height, and it has demonstrated a good capacity for identifying risk of CAD in women; 5 there are, however, no studies in the literature that compare these new indices in vegetarians and non-vegetarians.

Different DPs, and food choices may contribute to the development of diseases, and food choices may contribute to the development of diseases. In this study, we observed that the VEG and LOV groups had more balanced diets that were rich in fibers and adequate in terms of nutrients, 
Table 3 - Food consumption according to type of diet adopted

\begin{tabular}{|c|c|c|c|}
\hline Variable & VEG & LOV & OMNI \\
\hline Energy (kcal) & $1751.9(1348.7-2231.2)$ & $1431.2(1119.5-1861.6)$ & $1447.7(1155.7-1711.2)^{\mathrm{a}}$ \\
\hline Processed foods (\% TEV) & $8.8(0.4-14.4)$ & $5.7(0.0-19.8)$ & $14.9(5.1-22.3)$ \\
\hline Ultra-processed foods (\% TEV) & $2.7(0.0-9.3)$ & $0.0(0.0-4.2)$ & $6.3(0.0-24.4)^{b}$ \\
\hline Proteins (\% TEV) & $12.7(11.1-16.4)$ & $15.6(12.3-20.6)^{f}$ & $19.5(22.5-29.7)^{d}$ \\
\hline Carbohydrates (\% TEV) & $65.7(59.5-72.9)$ & $62.3(57.1-70.2)$ & $49.5(43.2-54.5)$ d.e \\
\hline Sugars (\% TEV) & $15.1(10.9-20.7)$ & $12.0(9.7-19.4)$ & $7.1(5.0-10.8)^{b . d}$ \\
\hline Dietary fiber $(\mathrm{g})$ & $42.5(34.7-52)$ & $35.1(26.9-45.7)$ & $13.8(11.9-22.3)^{\text {d.e }}$ \\
\hline Total fats (\% TEV) & $22.7(16.2-25.8)$ & $20.7(14.0-29.5)$ & $28.4(23.5-30.3)^{a . b}$ \\
\hline Saturated fatty acids (\% TEV) & $3.4(2.7-4.4)$ & $5.1(3.0-7.1)$ & $10.5(8.9-12.5)^{\text {d.e }}$ \\
\hline Monounsaturated fatty acids (\% TEV) & $5.0(3.0-7.5)$ & $3.1(1.7-5.5)$ & $6.2(4.1-7.6)^{b}$ \\
\hline Polyunsaturated fatty acids (\% TEV) & $2.3(1.0-3.3)$ & $2.4(1.6-3.3)$ & $1.7(1.1-2.3)$ \\
\hline Cholesterol (mg) & $10.5(0.0-23.2)$ & $25.0(10.1-64.9)^{c}$ & $216.9(158.9-272.7)^{\text {d.e }}$ \\
\hline Omega-6/omega-3 ratio & $3.9(1.6-8.1)$ & $4.3(2.1-8.2)$ & $5.5(4.2-8.4)$ \\
\hline Sodium (mg) & $1152(854.2-1705.1)$ & $1010.7(771.1-1479.3)$ & $1548.7(1154.7-2148.4)^{\text {a.b }}$ \\
\hline $\mathrm{Na} /$ Kcal ratio & $0.7(0.5-0.98)$ & $0.7(0.6-1.07)$ & $1.3(0.96-1.68)^{\text {d.e }}$ \\
\hline Folic acid (mcg) & $341.1(225.4-507)$ & $280.9(143.3-408.5)$ & $236.3(144.7-356.3)^{\text {a }}$ \\
\hline Vitamin A (IU) & 9958.8 (6016.9 - 12130.4) & $10230.8(4365.1$ - 13978.8) & 3141.5 (1579.6-9180.6) \\
\hline Vitamin $B_{12}(\mathrm{mcg})$ & $0.04(0.00-0.77)$ & $0.2(0.08-0.30)$ & $2.2(1.06-3.8)^{\text {d.e }}$ \\
\hline Vitamin C (mg) & $82.8(48.8-127.9)$ & 75.7 (41.1-159.2) & $20.4(12.0-66.1)^{\text {d.e }}$ \\
\hline Vitamin D (IU) & $0.0(0.0-0.2)$ & $1.6(0.1-4.8)^{c}$ & $9.6(5.9-25.7)^{\text {d.e }}$ \\
\hline Vitamin E (mg) & $2.8(1.5-5.6)$ & $1.3(0.97-2.2)$ & $1.1(0.7-1.8)^{c . d}$ \\
\hline Calcium (mg) & 339.1 (257.4-512.6) & $380.3(278.7-497.1)$ & $307.9(185.7-369.7)$ \\
\hline Iron (mg) & $15.4(11.8-18.8)$ & $12.7(9.8-18.3)$ & $11.3(8.9-14.7)^{a}$ \\
\hline Magnesium (mg) & $235.6(196.7-339.6)$ & $213.4(131.3-258.6)$ & $135.6(113.7-249.6)^{a}$ \\
\hline Potassium (mg) & $1969.6(1410.6-2615.5)$ & $1759.9(1309.1-2288)$ & $1308.3(947.1-1611.6)^{a . b}$ \\
\hline Zinc (mg) & $4.3(3.6-5.5)$ & $3.9(2.5-5.1)$ & $5.9(5.2-10.6)^{a}$ \\
\hline Beta-carotene (mg) & $4.2(1.6-6.3)$ & $3.4(2-6.2)$ & $1.4(0.3-2.3)^{\text {d.e }}$ \\
\hline Carotenoids (mg) & $987.4(519.7-1212.5)$ & $1015.6(366.3-1318.4)$ & $261(94.5-523.2)^{\text {d.e }}$ \\
\hline \multicolumn{4}{|c|}{$\begin{array}{l}\text { Values shown as median and interquartile range. Kruskal-Wallis test with post hoc Bonferroni to analyze significance level. Values considered statistically } \\
\text { significant: } p<0.05 .{ }^{a} p<0.05 \text { between OMNI and VEG; }{ }^{b} p<0.05 \text { between } O M N I \text { and } L O V ;{ }^{c} p<0.05 \text { between LOV and VEG; }{ }^{d} p \leq 0.0001 \text { between } \\
\text { OMNI and VEG; }{ }^{e} p \leq 0.0001 \text { between OMNI and LOV; }{ }^{p} p \leq 0.001 \text { between LOV and VEG. TEV: total energy value. }\end{array}$} \\
\hline
\end{tabular}

such as vitamins A, C, and E and carotenoids, and low in saturated fatty acids, cholesterol, and sodium. Bowman et al. ${ }^{34}$ also observed differences between the vegetarian and omnivorous DPs; the first was characterized by greater consumption of micronutrients and lower consumption of saturated fat and sodium.
Greater intake of fruits, vegetables, and legumes is associated with lower risk of CVD, acute myocardial infarction, cardiovascular mortality, non-cardiovascular mortality, and total mortality. This is because these foods contain antioxidants and polyphenols, such as vitamin C, vitamin E, and carotenoids, which can prevent lipid 
Table 4 - Spearman correlation between percentage of total energy value from ultra-processed foods and cardiovascular risk factors, by dietary group

\begin{tabular}{|c|c|c|c|c|c|c|}
\hline & \multicolumn{2}{|c|}{ VEG } & \multicolumn{2}{|c|}{ LOV } & \multicolumn{2}{|c|}{ OMNI } \\
\hline & @ & $p$ value & $\varrho$ & p value & @ & $p$ value \\
\hline BMI $\left(\mathrm{kg} / \mathrm{m}^{2}\right)$ & -0.123 & 0.473 & 0.240 & 0.193 & -0.082 & 0.661 \\
\hline Fat mass (\%) & -0.161 & 0.348 & 0.047 & 0.802 & -0.044 & 0.813 \\
\hline WHR & -0.18 & 0.917 & 0.083 & 0.657 & 0.137 & 0.463 \\
\hline $\mathrm{WC}(\mathrm{cm})$ & -0.019 & 0.193 & 0.113 & 0.546 & 0.116 & 0.534 \\
\hline $\mathrm{NC}(\mathrm{cm})$ & 0.033 & 0.847 & 0.121 & 0.516 & -0.216 & 0.243 \\
\hline VAI & -0.043 & 0.804 & 0.050 & 0.788 & 0.183 & 0.325 \\
\hline LAP & -0.023 & 0.894 & 0.116 & 0.534 & 0.131 & 0.482 \\
\hline $\mathrm{CI}$ & 0.049 & 0.777 & -0.075 & 0.690 & 0.297 & 0.105 \\
\hline ABSI & 0.174 & 0.310 & -0.303 & 0.098 & 0.237 & 0.200 \\
\hline BRI & -0.052 & 0.764 & 0.099 & 0.596 & 0.123 & 0.510 \\
\hline SBP (mmHg) & $0.439^{*}$ & 0.007 & 0.011 & 0.951 & -0.157 & 0.399 \\
\hline DBP (mmHg) & 0.178 & 0.299 & -0.096 & 0.607 & -0.299 & 0.103 \\
\hline Blood sugar (mg/dL) & $0.422^{*}$ & 0.010 & -0.015 & 0.934 & 0.040 & 0.829 \\
\hline Insulin $(\mathrm{mIU} / \mathrm{mL})$ & 0.003 & 0.988 & 0.066 & 0.748 & 0.002 & 0.993 \\
\hline HOMA-IR & 0.094 & 0614 & 0.086 & 0.675 & 0.017 & 0.935 \\
\hline Triglycerides (mg/dL) & 0.069 & 0.691 & 0.033 & 0.859 & 0.105 & 0.572 \\
\hline $\mathrm{TC}(\mathrm{mg} / \mathrm{dL})$ & 0.025 & 0.886 & -0.293 & 0.110 & -0.127 & 0.497 \\
\hline LDL-c (mg/dL) & -0.121 & 0.480 & $-0.456^{*}$ & 0.010 & -0.097 & 0.605 \\
\hline HDL-c (mg/dL) & 0.236 & 0.165 & -0.87 & 0.641 & -0.054 & 0.774 \\
\hline
\end{tabular}

oxidation in the arterial vessel walls, reduce blood pressure, and improve endothelial function, in addition to the fibers that are associated with reduced insulin, total cholesterol, and LDL. ${ }^{35}$ In contrast, saturated fatty acids influence the production of inflammatory cytokines and insulin resistance, and sodium increases blood pressure; consequently, increased consumption of these nutrients is associated with increased cardiovascular mortality. ${ }^{36-38}$

Although vegetarian diets are favorable to health, 8,39 they may often not bring health benefits in the event that they are composed of processed foods. ${ }^{11}$ We observed greater consumption of UPF in the OMNI group, in comparison with LOV. The VEG group, even though they had low consumption of UPF, had elevated sugar intake (> $10 \%$ of TEV). Silveira et al. ${ }^{13}$ studied 503 vegetarians (83.7\% women), and they observed that $60 \%$ consumed UPF and sugar-sweetened beverages; the frequency of excess daily intake of UPF ( $\geq 3$ times daily) and sugarsweetened beverages ( $\geq 3$ times daily) were $16 \%$ and $20 \%$, respectively. Furthermore, excessive consumption of UPF ( $\geq 3$ times daily) was independently associated with overweight. A recent cross-sectional study of the NutriNet-Santé cohort, conducted in France with 21,212 participants with different DP (omnivorous, pescovegetarian, vegetarian, and vegan), found that increased avoidance of foods of animal origin was associated with increased consumption of UPF, demonstrating that not 


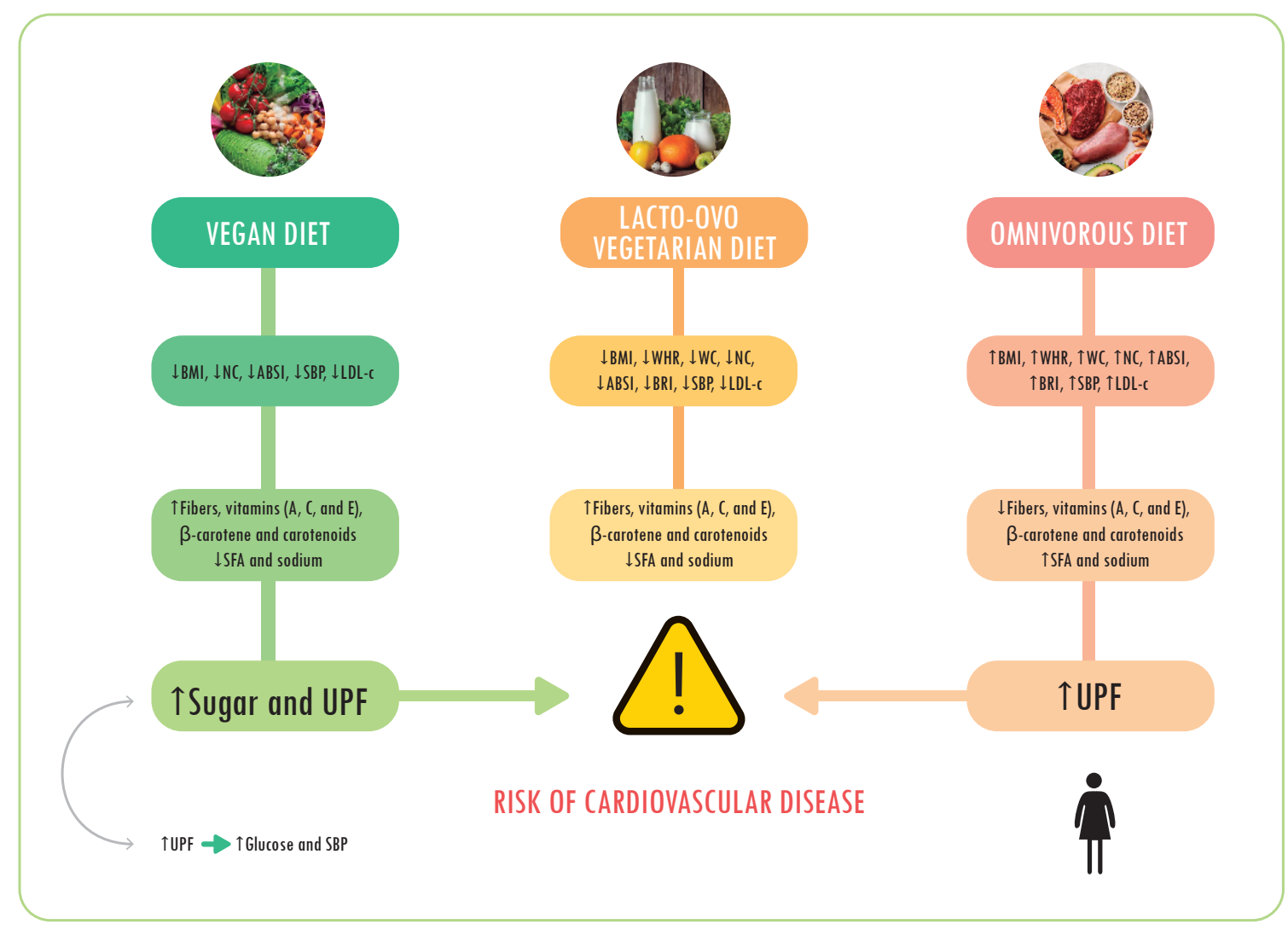

Figure 1 - Effect of dietary pattern on cardiovascular risk in women.

ABSI: body shape index; BMI: body mass index; BRI: body roundness index; LDL: low-density lipoprotein; NC: neck circumference; SBP: systolic blood pressure; SFA: saturated fatty acids; UPF: ultra-processed foods; WC: waist circumference; WHR: waist-to-height ratio.

all vegetarian diets necessarily bring health benefits, due to the potential effects of UPF.

We observed an association of UPF consumption with SBP and blood sugar in the VEG group. UPF are energy-dense, and they usually have higher total fat, saturated fat, sugar, and salt contents, as well as a lower amount of fiber and vitamins. High consumption of UPF is associated with increased prevalence of obesity, dyslipidemia, metabolic syndrome, and CVD. ${ }^{12,40}$ It is, accordingly, necessary to reduce consumption of these foods, regardless of the DP adopted.

Study limitations include the small number of volunteers, which can make it difficult to generalize the results. Participants were young, apparently healthy, and active; it is likely due to this that they did not show alterations in biochemical parameters and had low FRS scores. The lack of a tool to assess cardiovascular risk in young women is also a limitation in the current literature. Finally, the cross-sectional study design limits the capacity to establish a causal association, making it necessary to conduct prospective longitudinal studies in the future in order to confirm these results. Nonetheless, this was the first Brazilian study to characterize UPF consumption and associate it with cardiovascular risk factors in vegetarian women, comparing them with other DPs.

\section{Conclusion}

Women who adhered to vegetarian DP had better body composition and dietary quality than those with OMNI DP, suggesting that the former DP can confer benefits with respect to cardiovascular protection in young women. Nevertheless, future studies should consider consumption of UPF in vegetarians as a modifiable risk factor for CVD. 


\section{Author contributions}

Conception and design of the research: Oliveira BS, Aranha LN, Olivares PSG, Negrão TGR, Rosa G, Oliveira GMM. Acquisition of data: Oliveira BS, Aranha LN, Olivares PSG, Negrão TGR, Rosa G, Oliveira GMM. Analysis and interpretation of the data: Oliveira BS, Aranha LN, Olivares PSG, Negrão TGR, Rosa G, Oliveira GMM. Statistical analysis: Aranha LN, Rosa G, Oliveira GMM. Writing of the manuscript: Oliveira BS, Aranha LN, Olivares PSG, Negrão TGR, Rosa G, Oliveira GMM. Critical revision of the manuscript for intellectual content: Aranha LN, Rosa G, Oliveira GMM.

\section{Potential Conflict of Interest}

No potential conflict of interest relevant to this article was reported.

\section{References}

1. Roth GA, Mensah GA, Johnson CO, Addolorato G, Ammirati E, Baddour LM, et al. Global Burden of Cardiovascular Diseases and Risk Factors, 1990-2019: Update From the GBD 2019 Study. J Am Coll Cardiol. 2020;76(25):2982-3021. doi: 10.1016/j.jacc.2020.11.010

2. Arora S, Stouffer GA, Kucharska-Newton AM, Qamar A, Vaduganathan M, Pandey A, et al. Twenty Year Trends and sex Differences in Young Adults Hospitalized With Acute Myocardial Infarction. Circulation. 2019;139(8):1047-56. doi: 10.1161/CIRCULATIONAHA.118.037137.

3. Tabnet.datasus.gov [Internet]. Brasília: Ministério da Saúde; 2021 [cited 2021 Jan 05]. Available from: http://tabnet.datasus.gov.br/cgi/deftohtm. exe?sim/cnv/mat10uf.def.

4. Wilson PW, D'Agostino RB, Levy D, Belanger AM, Silbershatz H, Kannel WB. Prediction of Coronary Heart Disease Using Risk Factor Categories. Circulation. 1998;97(18):1837-47. doi: 10.1161/01.cir.97.18.1837.

5. Wang F, Chen Y, Chang Y, Sun G, Sun Y. New Anthropometric Indices or Old Ones: Which Perform Better in Estimating Cardiovascular Risks in Chinese Adults. BMC Cardiovasc Disord. 2018;18(1):14. doi: 10.1186/ s12872-018-0754-z.

6. Van Horn L, Carson JA, Appel LJ, Burke LE, Economos C, Karmally W, et al. Recommended Dietary Pattern to Achieve Adherence to the American Heart Association/American College of Cardiology (AHA/ACC) Guidelines: A Scientific Statement from the American Heart Association Circulation. 2016;134(22):505-29. doi: 10.1161/CIR.0000000000000462.

7. Rizzo NS, Jaceldo-Siegl K, Sabate J, Fraser GE. Nutrient Profiles of Vegetarian and Nonvegetarian Dietary Patterns. J Acad Nutr Diet. 2013;113(12):1610-9. doi: 10.1016/j.jand.2013.06.349.

8. Dinu M, Abbate R, Gensini GF, Casini A, Sofi F. Vegetarian, Vegan Diets and Multiple Health Outcomes: A Systematic Review With Meta-Analysis of Observational Studies. Crit Rev Food Sci Nutr 2017;57(17):3640-9. doi: 10.1080/10408398.2016.1138447.

9. Benatar JR, Stewart RAH. Cardiometabolic Risk Factors in Vegans; A Meta-Analysis of Observational Studies. PLoS One. 2018;13(12):e0209086 doi: 10.1371/journal.pone.0209086.

10. Kahleova H, Levin S, Barnard ND. Vegetarian Dietary Patterns and Cardiovascular Disease. Prog Cardiovasc Dis. 2018;61(1):54-61. doi 10.1016/j.pcad.2018.05.002.

\section{Sources of Funding}

There were no external funding sources for this study.

\section{Study Association}

This article is part of the thesis of master submitted by Bianca da Silva Oliveira, from Universidade Federal do Rio de Janeiro.

\section{Ethics approval and consent to participate}

This study was approved by the Ethics Committee of the Hospital Universitário Clementino Fraga Filho under the protocol number 89033118.1.0000.5257. All the procedures in this study were in accordance with the 1975 Helsinki Declaration, updated in 2013. Informed consent was obtained from all participants included in the study.

11. Satija A, Bhupathiraju SN, Spiegelman D, Chiuve SE, Manson JE, Willett W, et al. Healthful and Unhealthful Plant-Based Diets and the Risk of Coronary Heart Disease in U.S. Adults. J Am Coll Cardiol. 2017;70(4):41122. doi: 10.1016/j.jacc.2017.05.047.

12. Monteiro CA, Cannon G, Levy RB, Moubarac JC, Louzada ML, Rauber F, et al. Ultra-Processed Foods: What They are and How to Identify Them. Public Health Nutr. 2019;22(5):936-41. doi: 10.1017/S1368980018003762.

13. Silveira JAC, Meneses SS, Quintana PT, SANTOS VS. Association Between Overweight and Consumption of Ultra-Processed Food and Sugar-Sweetened Beverages Among Vegetarians. Rev. Nutri., Campinas, 30(4):431-41. doi: https://doi.org/10.1590/1678-98652017000400003.

14. Gehring J, Touvier M, Baudry J, Julia C, Buscail C, Srour B, et al. Consumption of Ultra-Processed Foods by Pesco-vegeTarians, Vegetarians, and Vegans: Associations with Duration and Age at Diet Initiation. J Nutr. 2021;151(1):120-31. doi: 10.1093/jn/nxaa196.

15. World Health Organization. Obesity: Preventing and Managing the Global Epidemic - Report of a WHO Consultation. Geneva: World Health Organization; 2000.

16. Associação Brasileira para o Estudo da Obesidade e da Síndrome Metabólica. Diretrizes Brasileiras de Obesidade: Associação Brasileira para o Estudo da Obesidade e da Síndrome Metabólica. São Paulo: ABESO; 2016.

17. Amato MC, Giordano C. Visceral Adiposity Index: an Indicator of Adipose Tissue Dysfunction. Int J Endocrinol. 2014;2014:730827. doi: $10.1155 / 2014 / 730827$.

18. Nascimento JXPT, Chein MBC, Sousa RML, Ferreira AS, Navarro PA, Brito LMO. Importance of lipid Accumulation Product Index as a Marker of CVD Risk in PCOS Women. Lipids Health Dis. 2015;14:62. doi: 10.1186/ s12944-015-0061-y.

19. Liu XZ, Qian JD, Li HH, Wang LJ, Wu MK, Wang Q, et al. Body Roundness Index is Significantly Associated with Prehypertension and Hypertension in Nonobese Chinese Subjects. Biomed Environ Sci. 2019;32(11):854-9. doi: 10.3967/bes2019.106.

20. Dhana K, Kavousi M, Ikram MA, Tiemeier HW, Hofman A, Franco OH. Body Shape Index in Comparison with other Anthropometric Measures in Prediction of Total And Cause-Specific Mortality. J Epidemiol Community Health. 2016;70(1):90-6. doi: 10.1136/jech-2014-205257. 
21. Lohman TG, Roche AF, Martorell R. Anthropometric Standardization Reference Manual. Champaign: Human Kinetics; 1988.

22. Malachias MVB, Gomes MAM, Nobre F, Alessi A, Feitosa AD, Coelho EB. 7th Brazilian Guideline of Arterial Hypertension: Chapter 2 - Diagnosis and Classification. Arq Bras Cardiol. 2016;107(3 Suppl 3):7-13. doi: 10.5935/abc. 20160152.

23. Friedewald WT, Levy RI, Fredrickson DS. Estimation of the Concentration of Low-Density Lipoprotein Cholesterol in Plasma, Without Use of the Preparative Ultracentrifuge. Clin Chem. 1972;18(6):499-502.

24. Matthews DR, Hosker JP, Rudenski AS, Naylor BA, Treacher DF, Turner RC. Homeostasis Model Assessment: Insulin Resistance and Beta-Cell Function from Fasting Plasma Glucose and Insulin Concentrations in Man. Diabetologia. 1985;28(7):412-9. doi: 10.1007/BF00280883.

25. Otten J, Helliwig JP, Meyers LD. The Dietary Reference Intakes: The Essential Guide to Nutrient Requirements. Washington: National Academies Press; 2006.

26. Précoma DB, Oliveira GMM, Simão AF, Dutra OP, Coelho OR, Izar MCO, et al. Atualização da Diretriz de Prevenção Cardiovascular da Sociedade Brasileira de Cardiologia - 2019. Arq Bras Cardiol. 2019; 113(4):787-891. doi: 10.5935/abc.20190204

27. Monteiro CA, Cannon G, Moubarac JC, Levy RB, Louzada MLC, Jaime PC. The UN Decade of Nutrition, the NOVA Food Classification and the Trouble with Ultra-Processing. Public Health Nutr. 2018;21(1):5-17. doi: 10.1017/S1368980017000234.

28. Matsudo M, Araújo T, Matsudo V, Andrade D, Andrade E, Oliveira LC, et al. Questionário Internacional de Atividade Física (IPAQ): Estudo de Validade e Reprodutibilidade no Brasil. Ativ. Física e Saúde. 2001;6(1):518. doi: https://doi.org/10.12820/rbafs.v.6n2p5-18

29. Navarro JCA, Antoniazzi L, Oki AM, Bonfim MC, Hong V, Bortolotto LA, et al. Prevalence of Metabolic Syndrome and Framingham Risk Score in Apparently Healthy Vegetarian and Omnivorous Men. Arq Bras Cardiol. 2018;110(5):430-7. doi: 10.5935/abc.20180073.

30. Brindle P, Beswick A, Fahey T, Ebrahim S. Accuracy and Impact of Risk Assessment in the Primary Prevention of Cardiovascular Disease: A Systematic Review. Heart. 2006;92(12):1752-9. doi: 10.1136/ hrt.2006.087932.

31. Freaney PM, Khan SS, Lloyd-Jones DM, Stone NJ. The Role of Sex-Specific Risk Factors in the Risk Assessment of Atherosclerotic Cardiovascular
Disease for Primary Prevention in Women. Curr Atheroscler Rep. 2020;22(9):46. doi: 10.1007/s11883-020-00864-6.

32. Jin Y, Kanaya AM, Kandula NR, Rodriguez LA, Talegawkar SA. Vegetarian Diets are Associated with Selected Cardiometabolic Risk Factors Among Middle-OLDER Aged South Asians in the United States. J Nutr. 2018;148(12):1954-60. doi: 10.1093/jn/nxy217.

33. Chiu YF, Hsu CC, Chiu TH, Lee CY, Liu TT, Tsao CK, et al. CrosssecTional and Longitudinal Comparisons of Metabolic Profiles between Vegetarian and non-Vegetarian Subjects: A Matched Cohort Study. Br J Nutr. 2015;114(8):1313-20. doi: 10.1017/S0007114515002937.

34. Bowman SA. A Vegetarian-Style Dietary Pattern is Associated with Lower Energy, Saturated Fat, and Sodium Intakes; and Higher Whole Grains, Legumes, Nuts, and Soy Intakes by Adults: National Health and Nutrition Examination Surveys 2013-2016. Nutrients. 2020;12(9):2668. doi: 10.3390/nu12092668.

35. Miller V, Mente A, Dehghan M, Rangarajan S, Zhang X, Swaminathan S, et al. Fruit, Vegetable, and Legume Intake, and Cardiovascular Disease and Deaths in 18 Countries (PURE): A Prospective Cohort Study. Lancet. 2017;390(10107):2037-49. doi: 10.1016/S0140-6736(17)32253-5.

36. Wang Q, Afshin A, Yakoob MY, Singh GM, Rehm CD, Khatibzadeh S, et al. Impact of Nonoptimal Intakes of Saturated, Polyunsaturated, and Trans Fat on Global Burdens of Coronary Heart Disease. J Am Heart Assoc. 2016;5(1):e002891. doi: 10.1161/JAHA.115.002891.

37. Kennedy A, Martinez K, Chuang CC, LaPoint K, McIntosh M. Saturated Fatty Acid-Mediated Inflammation and Insulin Resistance in Adipose Tissue: Mechanisms of Action and Implications. J Nutr. 2009;139(1):1-4. doi: 10.3945/jn.108.098269.

38. Otto MCO, Afshin A, Micha R, Khatibzadeh S, Fahimi S, Singh G, et al The Impact of Dietary and Metabolic Risk Factors on Cardiovascular Diseases and Type 2 Diabetes Mortality in Brazil. PLoS One. 2016;11(3):e0151503. doi: 10.1371/journal.pone.0151503.

39. Huang T, Yang B, Zheng J, Li G, Wahlqvist ML, Li D. Cardiovascular Disease Mortality and Cancer Incidence in Vegetarians: A Meta-Analysis and Systematic Review. Ann Nutr Metab. 2012;60(4):233-40. doi: $10.1159 / 000337301$

40. Srour B, Fezeu LK, Kesse-Guyot E, Allès B, Méjean C, Andrianasolo RM, et al. Ultra-Processed Food Intake and Risk of Cardiovascular Disease: Prospective Cohort Study (NutriNet-Santé). BMJ. 2019;365:11451. doi: 10.1136/bmj.11451. 